EDUCAONLINE

ISSN 1983-2664

EDUCOMUNICACÁO

EDUCAÇ̃o E NOVAS TECNOLOGIAS

Volume 7 - № 3 - Setembro/Dezembro de 2013

\title{
Formação de pesquisadores para uso do Mendeley: relato de experiência
}

\author{
Claudia Machado \\ Universidade do Minho - Braga, Portugal \\ actmachado@hotmail.com
}

\begin{abstract}
Resumo
No âmbito académico, várias tem sido as ferramentas que auxiliam o pesquisador na elaboração dos seus trabalhos. O Mendeley insere-se neste contexto, visto que possibilita a gestão, partilha, citação de referências, para além de ser uma rede de investigação online de gestão de artigos em que é possível a ligação a outros pesquisadores e a descoberta de tendências de trabalhos que estão a ser desenvolvidos na área a qual se tem interesse. Neste sentido, face ao desconhecimento de muitos pesquisadores sobre a existência de tais ferramentas e suas funcionalidades, nesta comunicação apresenta-se um relato de experiência da ação de formação "MENDELEY: Software académico para gestão e partilha de trabalhos científicos" para alunos de pós-graduação da Universidade do Minho (UMINHO).. As inscrições, gratuitas, foram realizadas online e os certificados foram enviados em formato PDF por e-mail aos participantes após a formação. Estiveram presentes na formação 11 pesquisadores com níveis de conhecimentos distintos sobre o Mendley. Com vistas a conhecer o perfil dos participantes, bem como o grau de satisfação em relação à formação recebida, utilizamos um questionário online. Os resultados apontam a importância desse tipo de ação de formação como um mecanismo de apoio ao trabalho dos pesquisadores.
\end{abstract}

Palavras-chave: Formação, académico, Mendeley, pesquisadores. 
Volume 7 - № 3 - Setembro/Dezembro de 2013

\title{
Training of Researchers to use Mendeley: experience report
}

\begin{abstract}
In the academic context, has been several tools that assist the researcher in the development of their work. The Mendeley fits into this context, since it enables the management, sharing, citation of references, in addition to being a research network management online articles in which it is possible to link to other researchers and the discovery of trends in jobs that are being developed in the area to which it has an interest. In this sense, due to the ignorance of many researchers about the existence of such tools and their features, this paper presents an experience report of the action training "Mendeley: Academic software for management and sharing of scientific works" for students in post-graduate of the University of Minho (UMINHO). The registration was free, were conducted online and certificates were sent in PDF format by email to participants after the training. Attended the training 11 researchers with different levels of knowledge about the Mendley. In order to know the profile of the participants, as well as the degree of satisfaction with the training received, we use an online questionnaire. The results show the importance of such training activities as a mechanism to support the work of researchers.
\end{abstract}

Keywords: Training, academic, Mendeley, researchers.

\section{Introdução}

A utilização das ferramentas da Web 2.0 tem-se tornado uma realidade incontornável, pela facilidade com que se publica, interage e colabora coletivamente, ou seja, passamos de utilizadores (sujeitos passivos) a produtores (sujeitos ativos) (MACHADO, 2008, 2009A, 2009B; MACHADO \& FARIAS, 2012; MACHADO, 2011).

Porém, no âmbito académico, apesar de existirem várias ferramentas da Web 2.0 que auxiliam o pesquisador na elaboração dos seus trabalhos, é ainda escassa a quantidade de pesquisas que as exploram (Neubert \& Rodrigues, 2012). Do universo 
EDUCAONLINE

ISSN 1983-2664

EDUCOMUNICACÁO

EDUCAḈ̃o E NOVAS TECNOLOGIAS

Volume 7 - № 3 - Setembro/Dezembro de 2013

das ferramentas Web 2.0 que ainda são pouco conhecidas e exploradas no âmbito académico, encontra-se o Mendeley. O Mendeley é um software académico gratuito que possibilita fazer a gestão, partilha, citação de referencias, para além de ser uma rede de investigação online de gestão de artigos em que é possível a ligação a outros pesquisadores e a descoberta de tendências de trabalhos que estão a ser desenvolvidos na área a qual se tem interesse.

A utilização do Mendeley exige aprendizagem, pois não é uma ferramenta tão intuitiva (VILICIC, 2012, p. 116). Neste sentido e face ao desconhecimento de muitos pesquisadores sobre a existência de tais ferramentas e suas funcionalidades, neste texto apresenta-se um relato de experiência da ação de formação "MENDELEY: Software académico para gestão e partilha de trabalhos científicos" organizado pela Professora Doutora Maria João Gomes e teve como público-alvo alunos de pósgraduação da UMINHO.

\title{
A Web 2.0 e o Mendeley
}

De acordo com o relatório intitulado "If you build it, will they come? How researchers perceive and use web 2.0", os próprios pesquisadores

\begin{abstract}
are the most important enablers and communicators of emerging best practice. It is important that they should consider the full range of available tools and services as an intrinsic part of the research and scholarly communication process, and seek to learn from each other about new developments and practices that prove beneficial. Where web 2.0 tools and services have proved useful, the researchers involved can play a valuable role in exchanging information, thereby increasing awareness of the range of available tools and services (generic and discipline specific) and their utility for particular activities and settings. (PROCTOR, WILLIAMS, \& STEWART, 2010, p. 53)
\end{abstract}

$\mathrm{Na}$ atual sociedade da informação e do conhecimento, segundo Victor Henning, o mentor do Mendeley, em uma entrevista concedida a revista Veja publicada em 19/12/2012, "o caminho óbvio é compartilhar essas informações e deixar que nossos

Laboratório de Pesquisa em Tecnologias da Informação e da Comunicação - LATEC/UFRJ 
EDUCAONLINE

ISSN $1983-2664$

EDUCOMUNICACÁ̃o

EDUCAḈ̃ E NOVAS TECNOLOGIAS

\section{Volume 7 - N 3 - Setembro/Dezembro de 2013}

cadastrados se comuniquem livremente uns com os outros." (VILICIC, 2012, p. 117). Neste sentido, nota-se que o Mendeley para além de possibilitar ao pesquisador a agilização na normalização do seu trabalho científico, permite a promulgação e disseminação das ideias, através de um sistema de compartilhamento científico.

O Mendeley foi criado em 2008 por, na época, 3 estudantes de doutoramento, e teve como ideia inicial criar um software que formatasse automaticamente tanto a tese, como os textos nos padrões exigidos pelas revistas científicas. Logo evoluiu para o site (www.mendeley.com) e atualmente possui mais de 2 milhões de membros, na sua maioria são estudantes de graduação ou pós graduação.

Para aceder a versão gratuita do Mendeley, em que é disponível 2Gb de armazenamento de arquivos online grátis, é necessário um navegador com ligação à internet, e criar uma conta de utilizador. Existe ainda, após a conta criada, a possibilidade de fazer download de uma versão (Mendeley Desktop) que funciona localmente com as mesmas funcionalidades disponíveis na versão gratuita na Web, mas faz a sincronização automática entre a plataforma Mendeley na Web e o Mendeley Desktop. (THE MENDELEY SUPPORT TEAM, 2011)

Recentemente, mais precisamente em março de 2013, os Serviços de Biblioteca, Informação Documental e Museologia (SBIDM) da Universidade de Aveiro (UA) elaborou um quadro comparativo entre o EndNote X5, Mendeley e EndNote Web (SBIDM, 2013). Face ao tema do nosso texto, reportaremos-nos de seguida somente às funcionalidades do Mendeley descritas no quadro da SBDIM (Figura 1), sem contudo suprimir a importância dos outros gerenciadores. 
EDUCAONLINE

ISSN 1983-2664

\section{Volume 7 - № 3 - Setembro/Dezembro de 2013}

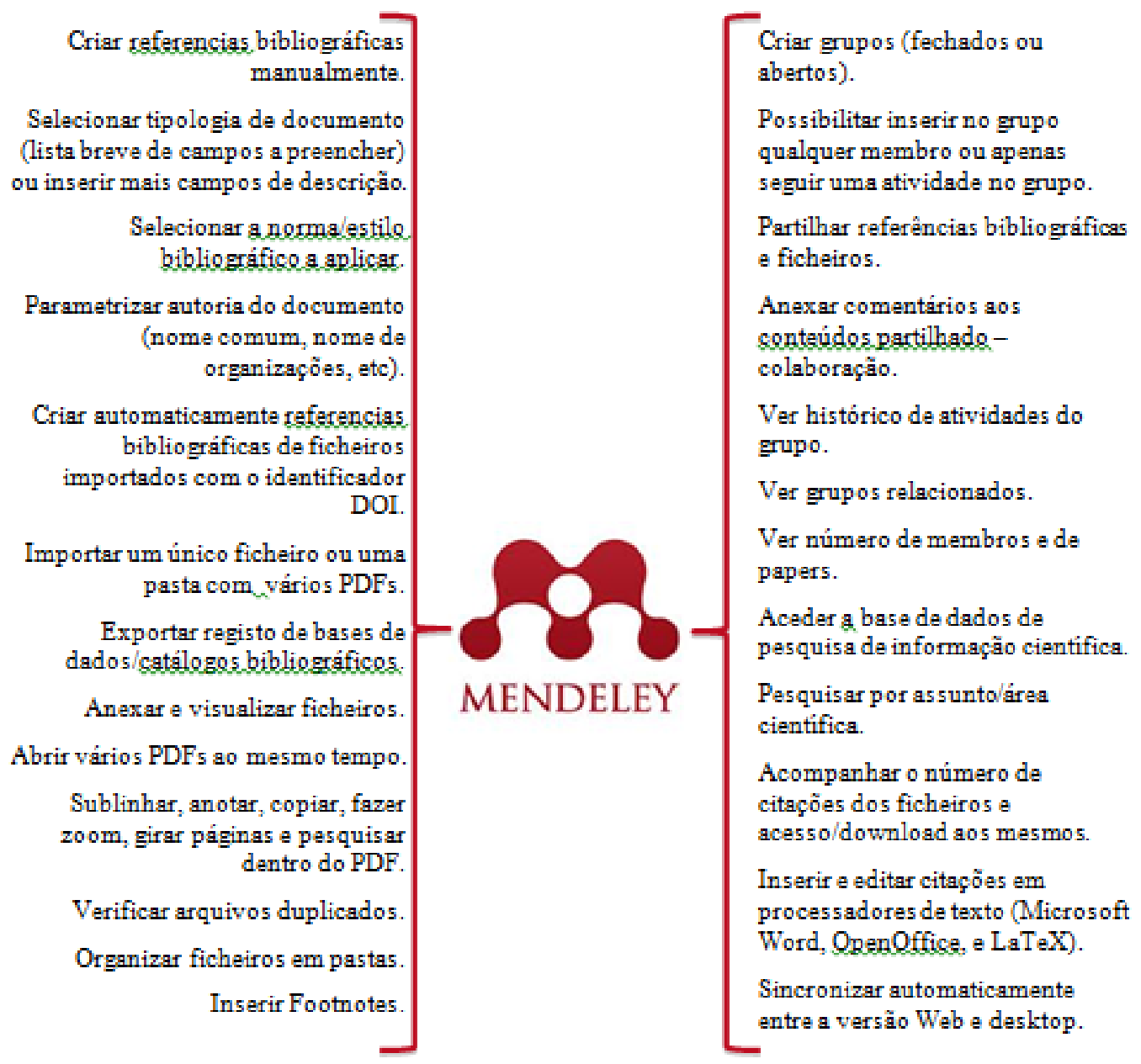

Figura 1: Mendeley e suas funcionalidade (SBIDM, 2013).

\section{Metodologia}

Este estudo em termos metodológicos, assumiu a forma de investigação-ação (IA) por objetivar, compreender e melhorar práticas em um determinado contexto (Bogdan \& Biklen, 1994; Coutinho, 2011). Sendo assim, a IA se torna adequada visto ao objetivo que era dar a conhecer aos pesquisadores o Mendeley e suas principais funcionalidades para que possam vir a utilizá-lo como um mecanismo de apoio aos seus trabalhos académicos.

O Workshop foi estruturado contemplando momentos teórico e prático. No que se refere ao momento teórico, este ocorreu nas primeiras $1 \mathrm{~h} 30$ e teve como objetivo 
EDUCAONLINE

ISSN 1983-2664

EDUCOMUNICACÁo

EDUCAḈ̃ E NOVAS TECNOLOGIAS

Volume 7 - № 3 - Setembro/Dezembro de 2013

dar a conhecer o Mendeley e as suas principais funcionalidades, conforme podemos observar de seguida:

- O que é o Mendeley;

- Conhecer as funcionalidades do Mendeley;

- Criar uma conta online;

- Instalar Mendeley Desktop;

- Criar a sua biblioteca pessoal;

- Organizar as suas referências;

- Citar as suas referências e criar bibliografias no Word;

- Colaborar em grupo no Mendeley.

Já no que se refere ao momento prático, este ocorreu nas últimas 1 h30 do Workshop e objetivou a prática, através de atividades, do que fora exposto no momento teórico. De entre as atividades listamos abaixo:

- Importar referência bibliográfica da Web;

- Importar referência bibliográfica a partir de um ficheiro em PDF;

- Sublinhar e fazer anotações num documento;

- Instalar o Web Importer;

- Inserir citação num documento em Word;

- Inserir bibliografia no documento Word;

- Pesquisar sobre grupos na área de interesse;

- Criar grupo de trabalho;

- Convidar pesquisadores/colaboradores;

- Partilhar artigo.

Para a realização da ação de formação criamos um folder (figura 2) e enviamos por email aos potenciais pós-graduandos. Para além disso, fizemos a impressão de 


\section{Volume 7 - № 3 - Setembro/Dezembro de 2013}

alguns folders e afixamos nas salas dos mestrandos e doutorandos do Instituto de Educação da UMINHO.

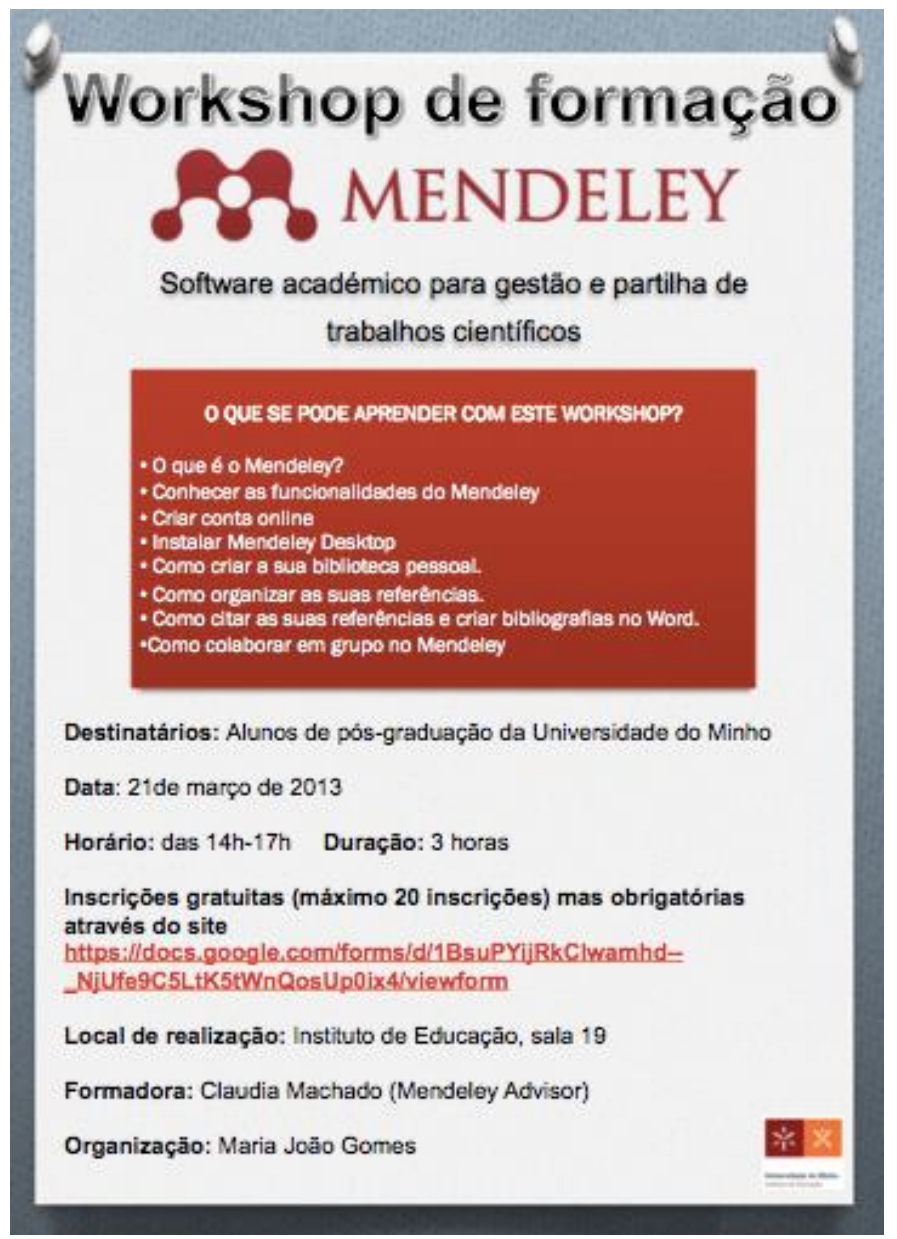

Figura 2: Folder de divulgação do workshop.

As inscrições eram gratuitas, mas obrigatórias, visto o limite de vagas disponíveis. Para se inscreverem era necessário aceder ao endereço do site que estava disponibilizado no folder e preencher os dados solicitados.

Após o workshop, os certificados foram enviados em formato PDF por e-mail aos participantes.

$\mathrm{Na} I \mathrm{~A}$, pode-se recorrer tanto às técnicas quantitativas quanto qualitativas. Neste estudo, utilizamos a observação e o questionário. No que se refere a observação, ajudou-nos a registar momentos do Workshop que serviram de subsídios na elaboração do questionário. 
EDUCAONLINE

ISSN $1983-2664$

EDUCOMUNICACÃo

EDUCAÇÃo E NOVAS TECNOLOGIAS

Volume 7 - N 3 - Setembro/Dezembro de 2013

O questionário teve caráter anônimo e confidencial (TUCKMAN, 2005) com perguntas abertas e fechadas. Foi implementado no serviço de elaboração do questionário online Qualtrics (www.qualtrics.com) por não exigir conhecimentos técnicos e ser uma ferramenta maleável (VAZ, RODRIGUES, LOUREIRO, BARBOSA, \& ANTUNES, 2009). A solicitação aos pesquisadores para responderem ao questionário foi enviada por e-mail, na qual se incluiu o link que dava acesso ao mesmo. O objetivo do questionário era conhecer o perfil dos pesquisadores, bem como o grau de satisfação em relação à ação de formação recebida.

Para a análise dos dados das questões fechadas recorremos ao tratamento estatístico associado ao serviço de elaboração do questionário online e realizamos análise de conteúdos para questões abertas.

O Workshop ocorreu nas dependências do Instituto de Educação da UMINHO em Braga-Portugal com duração de 3h, sendo dividido entre momento teórico (1h30) e prático (1h30). Inscreveram-se no Workshop 14 pós-graduandos, porém estiveram presentes na formação 11 e destes somente 10 responderam ao questionário.

De entre os pesquisadores que responderam ao questionário, 5 (50\%) são do sexo feminino e 5 (50\%) do sexo masculino. Relativamente à faixa etária, a maioria 7 (70\%) encontrava-se entre 36 e 46 anos, 2 (20\%) com idade superior a 47 anos e 1 (10\%) entre 25 a 35 anos de idade. A grande maioria 9 (90\%) fazia parte do Instituto da Educação e somente 1 (10\%) fazia parte da Escola de Direito da UMINHO. No que se refere à pós-graduação que estavam a realizar, a maioria 7 (70\%) respondeu que eram alunos de doutoramento e 3 (30\%) de mestrado.

\section{Apresentação e análise dos resultados}

Com a finalidade de conhecer o grau de satisfação em relação à ação de formação recebida, a primeira parte do questionário solicitava aos pós-graduandos que se posicionassem relativamente a um conjunto de perguntas, e as respostas foram sistematizadas na tabela 1. 
Volume 7 - № 3 - Setembro/Dezembro de 2013

Tabela 1: Conjunto de perguntas acerca do grau de satisfação em relação à formação recebida.

\begin{tabular}{|c|c|c|c|c|c|}
\hline Itens & 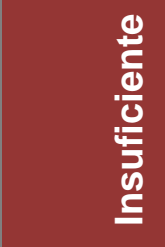 & $\frac{\text { के }}{\frac{5}{5}}$ & Eิ & 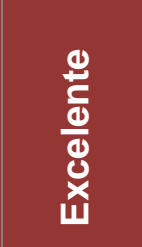 & 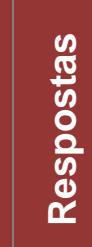 \\
\hline $\begin{array}{l}\text { 1. Ao apresentar-se neste Workshop } \\
\text { os seus conhecimentos sobre a } \\
\text { temática abordada eram? }\end{array}$ & $\begin{array}{l}6 \\
(60 \%)\end{array}$ & $\begin{array}{l}3 \\
(30 \%)\end{array}$ & $\begin{array}{l}0 \\
(0 \%)\end{array}$ & $\begin{array}{l}1 \\
(10 \%)\end{array}$ & 10 \\
\hline $\begin{array}{l}\text { 2. Este Workshop correspondeu a sua } \\
\text { expectativa? }\end{array}$ & $\begin{array}{l}0 \\
(0 \%)\end{array}$ & $\begin{array}{l}1 \\
(10 \%)\end{array}$ & $\begin{array}{l}2 \\
(20 \%)\end{array}$ & $\begin{array}{l}7 \\
(70 \%)\end{array}$ & 10 \\
\hline $\begin{array}{l}\text { 3. A formadora transmitiu com clareza } \\
\text { os assuntos abordados? }\end{array}$ & $\begin{array}{l}0 \\
(0 \%)\end{array}$ & $\begin{array}{l}0 \\
(0 \%)\end{array}$ & $\begin{array}{l}3 \\
(30 \%)\end{array}$ & $\begin{array}{l}7 \\
(70 \%)\end{array}$ & 10 \\
\hline $\begin{array}{l}\text { 4. A formadora conseguiu criar um } \\
\text { clima propício à participação? }\end{array}$ & $\begin{array}{l}0 \\
(0 \%)\end{array}$ & $\begin{array}{l}1 \\
(10 \%)\end{array}$ & $\begin{array}{l}3 \\
(30 \%)\end{array}$ & $\begin{array}{l}6 \\
(60 \%)\end{array}$ & 10 \\
\hline $\begin{array}{l}\text { 5. A formadora dominava o assunto } \\
\text { exposto? }\end{array}$ & $\begin{array}{l}0 \\
(0 \%)\end{array}$ & $\begin{array}{l}0 \\
(0 \%)\end{array}$ & $\begin{array}{l}2 \\
(20 \%)\end{array}$ & $\begin{array}{l}8 \\
(80 \%)\end{array}$ & 10 \\
\hline $\begin{array}{l}\text { 6. Os métodos utilizados foram os } \\
\text { mais adequados? }\end{array}$ & $\begin{array}{l}0 \\
(0 \%)\end{array}$ & $\begin{array}{l}2 \\
(20 \%)\end{array}$ & $\begin{array}{l}1 \\
(10 \%)\end{array}$ & $\begin{array}{l}7 \\
(70 \%)\end{array}$ & 10 \\
\hline $\begin{array}{l}\text { 7. Os objetivos do Workshop eram } \\
\text { claros? }\end{array}$ & $\begin{array}{l}0 \\
(0 \%)\end{array}$ & $\begin{array}{l}1 \\
(10 \%)\end{array}$ & $\begin{array}{l}2 \\
(20 \%)\end{array}$ & $\begin{array}{l}7 \\
(70 \%)\end{array}$ & 10 \\
\hline $\begin{array}{l}\text { 8. O Workshop estava bem } \\
\text { estruturado? }\end{array}$ & $\begin{array}{l}0 \\
(0 \%)\end{array}$ & $\begin{array}{l}1 \\
(10 \%)\end{array}$ & $\begin{array}{l}3 \\
(30 \%)\end{array}$ & $\begin{array}{l}6 \\
(60 \%)\end{array}$ & 10 \\
\hline $\begin{array}{l}\text { 9. A duração do Workshop, } \\
\text { relativamente ao seu conteúdo, foi } \\
\text { adequada? }\end{array}$ & $\begin{array}{l}1 \\
(10 \%)\end{array}$ & $\begin{array}{l}1 \\
(10 \%)\end{array}$ & $\begin{array}{l}3 \\
(30 \%)\end{array}$ & $\begin{array}{l}5 \\
(50 \%)\end{array}$ & 10 \\
\hline $\begin{array}{l}\text { 10. Como classifica os resultados } \\
\text { alcançados pela frequência deste } \\
\text { Workshop? }\end{array}$ & $\begin{array}{l}0 \\
(0 \%)\end{array}$ & $\begin{array}{l}1 \\
(10 \%)\end{array}$ & $\begin{array}{l}4 \\
(40 \%)\end{array}$ & $\begin{array}{l}5 \\
(50 \%)\end{array}$ & 10 \\
\hline
\end{tabular}

A análise do item 1 da tabela 1 , permite destacar que os conhecimentos dos pesquisadores sobre o Mendeley eram na sua grande maioria $9(90 \%)$ "insuficiente" ou "regular" e apenas 1 (10\%) considerar ter conhecimento "excelente". Relativamente à pergunta que tratava se o workshop tinha correspondido as suas expectativas (item 2), a grande maioria 9 (90\%) respondeu positivamente a esta questão e somente $1(10 \%)$ indicou "regular". 


\section{Volume 7 - № 3 - Setembro/Dezembro de 2013}

Analisando os itens 3 a 5 que abordam questão sobre a formadora, verifica-se que a totalidade 10 (100\%) dos pesquisadores acharam que os assuntos abordados foram transmitidos com clareza sinalizando ter sido "excelente" ou "bom", o que é reforçado pelo facto de 10 (100\%) acharem que foi "excelente" ou "bom" o domínio pela formadora do assunto exposto. Quanto a pergunta "A formadora conseguiu criar um clima propício à participação?", 9 (90\%) manifestaram "excelente" ou "bom", enquanto somente $1(10 \%)$ indicou que foi "regular".

O item 6 reportava-se à adequação dos métodos utilizados e da sua análise resulta claramente que foram considerados "excelente" ou "bom" pela grande maioria dos pesquisadores $(8-80 \%)$.

Quando perguntados se os objetivos do Workshop eram claros (item 7), os pesquisadores revelaram um alto nível de concordância, com 9 (90\%) das respostas a indicar "excelente" ou "bom". No que se refere à estrutura do Workshop, verifica-se através da análise do item 8 , que $9(90 \%)$ dos pesquisadores consideraram que foi "excelente" ou "bom". Verifica-se também através da análise do item 9, que 8 (80\%) dos pesquisadores consideram que foi "excelente" ou "bom" a duração do Workshop em relação ao conteúdo, sendo que $2(20 \%)$ dos pesquisadores consideram que foi "insuficiente" ou "regular".

Quanto à classificação dos resultados alcançados pela frequência do Workshop (item 10), a grande maioria 9 (90\%) indicou "excelente" ou "bom".

Uma análise global da tabela 1 indica que os resultados alcançados pela frequência do Workshop foram na sua grande maioria muito bons. O que se constata pelo facto da grande maioria dos pesquisadores sinalizarem que os métodos, os objetivos, a estrutura e a duração do Workshop estavam adequados para além da formadora ter: (i) transmitido com clareza os assuntos abordados; (ii) criado um clima propício à participação; e (iii) dominar o assunto exposto.

De seguida, procuramos conhecer os principais "pontos fortes" e "pontos fracos" do Workshop, e foram obtidas as respostas que se apresentam na tabela 2. 
Volume 7 - № 3 - Setembro/Dezembro de 2013

Tabela 2: Principais "pontos fortes" e "pontos fracos" do Workshop.

\begin{tabular}{|c|c|}
\hline Principais "pontos fortes" do Workshop & $\begin{array}{l}\text { Principais "pontos fracos" do } \\
\text { Workshon }\end{array}$ \\
\hline \multicolumn{2}{|l|}{ Transcrição das respostas } \\
\hline $\begin{array}{l}\text { Pesquisador 2: Conseguir a otimização } \\
\text { de um recurso importante para quem está }\end{array}$ & $\begin{array}{l}\text { Pesquisador 2: Pouco tempo para } \\
\text { explorar o instrumento em causa. }\end{array}$ \\
\hline $\begin{array}{l}\text { Pesquisador 3: Disseminação da } \\
\text { ferramenta de forma voluntária; a } \\
\text { excelência da ferramenta; a pertinência da } \\
\text { abordagem; tratar-se de acesso gratuito - } \\
\text { tanto o workshop quanto o Mendeley; a } \\
\text { disposição e abertura da formadora. }\end{array}$ & $\begin{array}{l}\text { Pesquisador 3: muitas pessoas, de } \\
\text { forma que de longe era difícil visualizar o } \\
\text { conteúdo dos slides; para gente que } \\
\text { como eu desconhecia por completo o } \\
\text { Mendeley, seria necessário um ritmo } \\
\text { significativamente mais tranquilo, com } \\
\text { um tempo para compreender de fato o } \\
\text { que se estava a fazer. A pressa cria } \\
\text { nreccãn Fa7-ce com annnriar-cs do }\end{array}$ \\
\hline $\begin{array}{l}\text { Pesquisador 4: Explicação individual das } \\
\text { dúvidas. }\end{array}$ & $\begin{array}{l}\text { Pesquisador 4: Muitas pessoas falando } \\
\text { ao mesmo tempo para sanar suas }\end{array}$ \\
\hline $\begin{array}{l}\text { Pesquisador 5: Compreender os } \\
\text { processos de referenciação do sofware. }\end{array}$ & Pesquisador 5: Tempo muito curto \\
\hline $\begin{array}{l}\text { Pesquisador 6: Foi adquirir } 0 \\
\text { conhecimento necessário para usar este }\end{array}$ & $\begin{array}{l}\text { Pesquisador 6: Constante volta da } \\
\text { formadora ao assunto por conta dos }\end{array}$ \\
\hline Pesquisador 7: Atenção e interatividade & Pesquisador 7: condução do grupo \\
\hline Pesquisador 8: referencia bibliografica & - \\
\hline
\end{tabular}

Da análise da tabela 2, nomeadamente no que se refere aos "pontos fortes" encontrados no Workshop, os pesquisadores 2, 5, 6 e 8 revelam a importância de conhecer este recurso para quem está a fazer investigação. Os pesquisadores 3, 4 e 7 referem-se à atenção e disposição para esclarecimento individualizado das dúvidas por parte da formadora.

Ainda no que se refere à tabela 2, quanto aos "pontos fracos", os pesquisadores 2, 3 e 5 relatam que precisam de mais tempo para explorar a ferramenta. Os pesquisadores 4, 6 e 7 sinalizam que faltou a melhor condução do grupo por parte da formadora.

Do conjunto de respostas dos pesquisadores à tabela 2, fica a perceção de certo consenso relativamente ao reconhecimento da importância do conhecimento da ferramenta Mendeley para quem está a fazer investigação e que esse tipo de ação 


\section{Volume 7 - № 3 - Setembro/Dezembro de 2013}

de formação precisa de mais tempo. Nota-se também que, apesar de sinalizarem positivamente à atenção e disposição da formadora para esclarecimento individualizado das dúvidas, também referiram que faltou melhor condução do grupo por parte da mesma, daí pode-se concluir a necessidade de ofertar ações de formações que considere os diferentes níveis de conhecimento do público-alvo sobre a ferramenta Mendeley.

Visando a melhoria das futuras edições do Workshop, procurou-se conhecer as sugestões dos pesquisadores (Tabela 3 ).

\section{Tabela 3: Sugestões para as futuras edições do Workshop}

\section{Sugestões de melhoria gostaria de fazer}

\section{Transcrição das respostas}

Pesquisador 2: Uma nova edição, se possível mais alargada.

Pesquisador 3: Além das sugeridas anteriormente, criar um núcleo, com alguns horários semanais, para auxiliar quem se inicia no Mendeley. Isto faria toda a diferença para efetivar sua utilização. Deixo meu agradecimento à Pesquisador 4: Curso com menos pessoas.

Pesquisador 5: para que a oficina pode ser dada em duas etapas: iniciante e Pesquisador 6: Que nos próximos Workshop sobre este assunto, houvesse um monitor para dividir a tarefa de explicar os que ficam atrasados com relação Pesquisador 7: estabelecer um roteiro coletivo que deve ser seguido por todos Pesquisador 8: tempo de formação.

$\mathrm{Da}$ análise das respostas dos pesquisadores à tabela 3, no que concerne as sugestões de melhoria para as futuras edições do Workshop, constata-se a necessidade de mais tempo para esse tipo de ação de formação (pesquisadores $2 \mathrm{e}$ 8), o que já corrobora com os "pontos fracos" indicados na tabela 2 pelos pesquisadores 2, 3, e 5. Para além disso, foi também ressaltado a importância de haver cursos diferenciados aos diferentes níveis de conhecimento sobre a ferramenta, como se referem os pesquisadores 5 e 6 . 
EDUCAONLINE

ISSN $1983-2664$

EDUCOMUNICACÁO

EDUCAÇÃo E NOVAS TECNOLOGIAS

Volume 7 - № 3 - Setembro/Dezembro de 2013

Uma última pergunta procurava conhecer se os pesquisadores indicariam este Workshop a outras pessoas e a totalidade 10 (100\%) sinalizou positivamente a esta questão. O que vem a ser corroborado com as respostas da tabela 1 (itens 2 a 10) e com as respostas aos "pontos fortes" da tabela 2.

\section{Considerações finais}

Este estudo permitiu revelar que este tipo de ação de formação que trata das ferramentas para que auxiliam o pesquisador no seu trabalho académico, torna-se de fundamental importância, visto que existe um desconhecimento por parte dos mesmos sobre a existência de tais ferramentas e suas funcionalidades.

Podemos também verificar que com a experiência realizada os pesquisadores ficaram satisfeitos e empolgados para utilizarem na sua prática pesquisadora 0 Mendeley não só para gerir, partilhar, citar referências, mas também utilizá-lo como rede de investigação online de gestão de artigos em que é possível ligar-se a outros pesquisadores e descobrir tendências de trabalhos que estão a ser desenvolvidos na área de seu interesse.

Das sugestões e indicações de "pontos fracos" do Workshop apresentadas pelos pesquisadores, nomeadamente ao que se refere à carga horária, quantidade de pessoas e cursos direcionados aos diferentes níveis de conhecimento sobre a ferramenta, serão considerados nas próximas edições neste tipo de ação de formação.

Nunca é demais lembrar que, para se realizar uma ação de formação que contemple no seu planeamento momentos práticos, é necessário verificar a infraestrutura disponível, tais como: acesso à Internet e computadores com possibilidade de instalação de softwares.

Face aos resultados obtidos, acreditamos que o objetivo foi atingido, ou seja, dar a conhecer aos pesquisadores o Mendeley e suas principais funcionalidades para que 
EDUCAONLINE

ISSN 1983-2664

EDUCOMUNICACÄO

EDUCAÇ̇̃o E NOVAS TECNOLOGIAS

Volume 7 - № 3 - Setembro/Dezembro de 2013

possam vir a utilizá-lo como um mecanismo de apoio aos seus trabalhos académicos.

\section{Referências}

BOGDAN, R., \& Biklen, S. Investigação qualitativa em educação. Uma Introdução à teoria e aos métodos. (Porto Editora, Ed.). Porto, 1994.

COUTINHO, C. P.. Metodologia de Investigação em Ciências Sociais (p. 343), . Almedina, 2011.

MACHADO, A. C. Novas formas de producão de conhecimento: utilizacão de ferramentas da web 2.0 como recurso pedagógico. Revista Udesc Virtu@।, 1(2), $2008 . \quad$ Disponível em: http://revistas.udesc.br/index.php/udescvirtual/article/view/1655/0. Acesso em: $15 / 05 / 2013$

MACHADO, A. C. Google Docs \& Spresdsheets: autoria colaborativa na web 2.0. Revista e-TEC, 2(1), 2009a. Disponível em: http://revistas.unibh.br/index.php/dtec/article/view/450/0. Acesso em: 15/05/2013

Machado, A. C. A ferramenta Google Docs: construção do conhecimento através da interação e colaboração. Revista Paidéi@, 2(1), 2009b. Disponível em:

http://revistapaideia.unimesvirtual.com.br/index.php?journal=paideia\&page=articl e\&op=view\&path\%5B\%5D=73. Acesso em: 15/03/2013

MACHADO, C. E-questionários: utilizando o Qualtrics Research Suite no contexto académico. XI Congresso SPCE. Guarda, 2011.

MACHADO, C., \& Farias, M. A. de A. Das teorias pré-tecnológicas às abordagens colaborativas. II Congresso Internacional TIC e Educação (pp. 409-418). Lisboa, 2012.

NEUBERT, P. da S., \& Rodrigues, S. R. Pesquisa bibliográfica e web 2.0: percepção de estudantes de pós-graduaçao em Ciência da Informaçao. 
EDUCAONLINE

ISSN 1983-2664

EDUCOMUNICACÁo

EDUCACÃo E NOVAS TECNOLOGIAS

Volume 7 - N 3 - Setembro/Dezembro de 2013

Informação \& Sociedade: Estudos, 22(3), 143-154, 2012. Disponível em: http://www.ies.ufpb.br/ojs/index.php/ies/article/view/10021. Acesso em: Acesso em: 01/3/2013

PROCTOR, R., Williams, R., \& Stewart, J. If you build it, will they come? How researchers perceive and use web 2.0. London, 2010. Disponível em: http://www.rin.ac.uk/system/files/attachments/web_2.0_screen.pdf. Acesso em $23 / 05 / 2013$

SBIDM. Gerir referências bibliográficas: Quadro comparativo EndNote X5, Mendeley e EndNote Web. Serviços de Biblioteca, Informação Documental e Museologia (SBIDM) - Universidade de Aveiro, 2013. Disponível em: www.ua.pt/ReadObject.aspx?obj=28263. Acesso em: 20/4/2013

THE MENDELEY SUPPORT TEAM. (2011). Getting Started with Mendeley. Mendeley Desktop. London: Mendeley Ltd, 2011. Disponível em: http://www.mendeley.com. Acesso em: 20/01/2013

TUCKMAN, B. W. Manual de Investigação em Educação: Metodologia para conceber e realizar o processo de investigação científica. (Serviço de Educacão/Fundação Calouste Gulbenkian, Ed.) (3a ed.), Lisboa, 2005.

VAZ, C., Rodrigues, M. do R., Loureiro, A., Barbosa, I., \& Antunes, P. Técnicas de recolha de dados em investigação qualitativa. Anais do Simpósio Internacional de Informática Educativa, 2009. Disponível em: http://www.academia.edu/504731/Tecnicas_de_recolha_de_dados_em_investig acao_qualitativa. Acesso em:10/01/2013

VILICIC, F. O Facebook dos cientistas. Revista Veja, 116-117, 2012. 
EDUCAONLINE

Volume 7 - № 3 - Setembro/Dezembro de 2013

\section{Sobre a Autora}

\begin{tabular}{|l|l|}
\hline & $\begin{array}{l}\text { Claudia Machado } \\
\text { Doutoranda em Educação - Tecnologia Educativa pela } \\
\text { Universidade do MINHO (2011 - Atual). Mestre em } \\
\text { Educação - Tecnologia Educativa pela Universidade do } \\
\text { MINHO (2011). Especialista em Educação a Distância } \\
\text { pelo SENAC (2007) e em Supervisão Escolar pela } \\
\text { UNIVERSO (1999). Licenciada em Pedagogia pelo } \\
\text { UNICEUMA (1997). Bolsista de Investigação da } \\
\text { Fundação para a Ciência e .a Tecnologia - Portugal. } \\
\text { (2012- atual). Mendeley Advisor. }\end{array}$ \\
\hline
\end{tabular}

Revista EducaOnline, Volume 7, № 3, Setembro/Dezembro de 2013. ISSN: 1983-2664. Este artigo foi submetido para avaliação em 18/06/2013 e aprovado para publicação em 21/07/2013. 